\title{
SYNCHRONIZATION AND CONTROL OF A NETWORK OF COUPLED REACTION-DIFFUSION SYSTEMS OF GENERALIZED FITZHUGH-NAGUMO TYPE*
}

\author{
Benjamin Ambrosio And M.A. Aziz-Alaoui ${ }^{1}$
}

Résumé. Nous considérons un réseau de systèmes de réaction-diffusion de type FitzHugh-Nagumo généralisés. Nous nous intéressons au comportement asymptotique et à la synchronisation du réseau. Ces résultats nous permettent d'étendre d'autres résultats obtenus pour un type particulier de systèmes de FitzHugh-Nagumo.

\begin{abstract}
We consider a network of reaction diffusion systems of generalized FitzHugh-Nagumo type, where the cubic function is replaced by a polynomial function with odd degree. We deal with asymptotic behaviour and synchronization of the whole network. These results extend a previous work in which we considered particular systems of FitzHugh Nagumo type.
\end{abstract}

\section{Introduction}

Let us denote by $w_{t}$ the time derivative of the function $w$. The FitzHugh-Nagumo model,

$$
\left\{\begin{array}{l}
x_{t}=c(F(x)+y+z) \\
y_{t}=\frac{1}{c}(x-a+b y)
\end{array}\right.
$$

where $F$ is a cubic function with positive leading coefficient, $z$ constant, and $a, b, c>0$, is a simplification of the well known Hodgkin-Huxley model describing the propagation of action potential in neurons, see for example, $[1-3,5,7]$. In [9], we considered a network of coupled reaction-diffusion systems of the following FitzHugh-Nagumo type $(\mathrm{FHN})$,

$$
\left\{\begin{aligned}
\epsilon u_{t} & =f(u)-v+d_{u} \Delta u \\
v_{t} & =u-\delta v+d_{v} \Delta v
\end{aligned}\right.
$$

where,

$$
f(u)=-u^{3}+3 u
$$

\footnotetext{
* This work was supported by Region Haute Normandie, France, and FEDER-RISC.

${ }^{1}$ LMAH, FR-CNRS-3335,

Université de Le Havre

ISCN

PRES Normandie Université,

BP 540, 76058, Le Havre Cedex,

FRANCE
} 
and where $\epsilon, \delta>0$, are small parameters. In this case, the underlying (ODE) part of system (1) induces an asymptotic evolution to a unique limit cycle for the trajectories diferent from $(0,0)$. We showed some results on asymptotic behaviour and synchronization for the network. Here, we will generalize some of these results. Let us consider a network of coupled reaction diffusion systems of the following generalized FHN type,

$$
\left\{\begin{aligned}
\epsilon u_{t} & =f(u)-v+d_{u} \Delta u+\gamma \\
v_{t} & =a u-b v+d_{v} \Delta v+\mu
\end{aligned}\right.
$$

where,

$$
f(u)=\sum_{k=1}^{p} d_{k} u^{k}
$$

is a polynomial function of odd degree with negative leading coefficient, $d_{p}<0, p \geq 3$. The parameter $\epsilon>0$ is small. The underlying (ODE) part of system (1) can induce a very more complicated asymptotic behaviour. We assume that $a, b, d_{u}$ are positive, and $d_{v}$ is non negative. We look for solutions $u=u(x, t), v=v(x, t)$ on a smooth bounded domain $\Omega \subset \mathbb{R}^{n}$, with zero-flux Neumann boundary conditions on the boundary of $\Omega$ :

$$
\frac{\partial u}{\partial \nu}=\frac{\partial v}{\partial \nu}=0,
$$

where $\nu$ denotes the exterior normal to the boundary. The coupling is chosen such that, for all $i=2, \ldots, N$, subsystem $\left(u_{i-1}, v_{i-1}\right)$ drives subsystem $\left(u_{i}, v_{i}\right)$. This means that the whole system reads as,

$$
\left\{\begin{aligned}
\epsilon u_{1 t} & =f\left(u_{1}\right)-v_{1}+d_{u_{1}} \Delta u_{1}+\gamma_{1} \\
v_{1 t} & =a_{1} u_{1}-b_{1} v_{1}+d_{v_{1}} \Delta v_{1}+\mu_{1} \\
\vdots & \\
\epsilon u_{i t} & =f\left(u_{i}\right)-v_{i}+d_{u_{i}} \Delta u_{i}+\alpha_{i}\left(u_{i-1}-u_{i}\right)+\gamma_{i} \\
v_{i t} & =a_{i} u_{i}-b_{i} v_{i}+d_{v_{i}} \Delta v_{i}+\beta_{i}\left(v_{i-1}-v_{i}\right)+\mu_{i} \\
\vdots & \\
\epsilon u_{N t} & =f\left(u_{N}\right)-v_{N}+d_{u_{N}} \Delta u_{N}+\alpha_{N}\left(u_{N-1}-u_{N}\right)+\gamma_{N} \\
v_{N t} & =a_{N} u_{N}-b_{N} v_{N}+d_{v_{N}} \Delta v_{N}+\beta_{N}\left(v_{N-1}-v_{N}\right)+\mu_{N}
\end{aligned}\right.
$$

where $\alpha_{i}, \beta_{i} \geq 0$, for $i=2, \ldots, N$.

\section{Analytical Results}

\subsection{Space homogeneous asymptotic behaviour}

Let $(u, v)$ be the solution of system (2), then we have the following result,

Théorème 2.1. Let,

$$
M=\sup _{x \in \mathbb{R}} f^{\prime}(x),
$$

and $\lambda$ be the smallest non zero eigenvalue of the Laplacian operator $(-\Delta)$ with zero flux Neumann boundary conditions. If,

$$
M-\lambda d_{u}<0
$$

then,

$$
\lim _{t \rightarrow+\infty}\left(\|\left(u-\bar{u}\left\|_{L^{2}(\Omega)}+\right\| v-\bar{v} \|_{L^{2}(\Omega)}\right)=0\right.
$$


where,

$$
\bar{u}(t)=\frac{\int_{\Omega} u(x, t) d x}{|\Omega|}, \quad \bar{v}(t)=\frac{\int_{\Omega} v(x, t) d x}{|\Omega|} .
$$

Moreover, $\bar{u}, \bar{v}$ are solutions of the following system,

$$
\left\{\begin{aligned}
\epsilon \bar{u}_{t} & =f(\bar{u})-\bar{v}+\gamma+g(t) \\
\bar{v}_{t} & =a \bar{u}-b \bar{v}+\mu
\end{aligned}\right.
$$

where $g(t)$ is a function going to 0 with exponential rate when $t$ goes to $+\infty$.

Démonstration. Let,

$$
\phi(t)=\frac{1}{2}\left(a \epsilon \int_{\Omega}|\nabla u|^{2}+\int_{\Omega}|\nabla v|^{2}\right)
$$

then,

$$
\begin{aligned}
\dot{\phi} & =\int_{\Omega}\left(\epsilon a \nabla u \nabla u_{t}+\nabla v \nabla v_{t}\right) \\
& =\int_{\Omega}\left(a \nabla u \nabla\left(f(u)-v+d_{u} \Delta u\right)+\nabla v \nabla\left(a u-b v+d_{v} \Delta v\right)\right) \\
& =\int_{\Omega}\left(a\left(f^{\prime}(u)|\nabla u|^{2}-d_{u}(\Delta u)^{2}\right)-b|\nabla v|^{2}-d_{v}(\Delta v)^{2}\right)
\end{aligned}
$$

Now, we use the following spectral property of laplacian operator with zero-flux Neumann boundary conditions, see for example [10],

$$
\int_{\Omega}(\Delta u)^{2} \geq \lambda \int_{\Omega} \nabla|u|^{2} .
$$

Then,

$$
\begin{aligned}
\dot{\phi} & \leq a\left(\int_{\Omega} M|\nabla u|^{2}-\lambda d_{u} \int_{\Omega}|\nabla u|^{2}\right)-b \int_{\Omega}|\nabla v|^{2}-\lambda d_{v} \int_{\Omega}|\nabla v|^{2} \\
& \leq a\left(M-\lambda d_{u}\right) \int_{\Omega}|\nabla u|^{2}-\left(\lambda d_{v}+b\right) \int_{\Omega}|\nabla v|^{2} .
\end{aligned}
$$

Now, since $\lambda d_{u}>M$ we have,

$$
\dot{\phi} \leq-2 \min \left(\frac{\lambda d_{u}-M}{\epsilon}, \lambda d_{v}+b\right) \phi,
$$

and thus,

$$
\phi(t) \leq \phi(0) e^{-c_{1} t}
$$

where,

Furthermore,

$$
c_{1}=2 \min \left(\frac{\lambda d_{u}-M}{\epsilon}, \lambda d_{v}+b\right) .
$$

$$
\begin{aligned}
\|u-\bar{u}\|_{\mathrm{L}^{2}(\Omega)}^{2}+\|v-\bar{v}\|_{\mathrm{L}^{2}(\Omega)}^{2} & \leq \frac{1}{\lambda}\left(\int_{\Omega}|\nabla u|^{2}+\int_{\Omega}|\nabla v|^{2}\right) \\
& \leq \frac{2}{\lambda} \max \left(\frac{1}{a \epsilon}, 1\right) \phi(t)
\end{aligned}
$$


which implies (5). In the remaining of the proof, we show that $\bar{u}$ et $\bar{v}$ are solutions of (6). We have,

$$
\left\{\begin{aligned}
\epsilon \bar{u}_{t} & =\frac{1}{|\Omega|} \int_{\Omega} f(u)-\bar{v}+\gamma \\
\bar{v}_{t} & =a \bar{u}-b \bar{v}+\mu
\end{aligned}\right.
$$

thus,

$$
\left\{\begin{aligned}
\epsilon \bar{u}_{t} & =\frac{1}{|\Omega|} \int_{\Omega}(f(u)-f(\bar{u}))+f(\bar{u})-\bar{v}+\gamma \\
\bar{v}_{t} & =a \bar{u}-b \bar{v}+\mu
\end{aligned}\right.
$$

Let us denote,

Then, we obtain :

$$
g(t)=\frac{1}{|\Omega|} \int_{\Omega}(f(u)-f(\bar{u}))
$$

$$
\left\{\begin{aligned}
\epsilon \bar{u}_{t} & =g(t)+f(\bar{u})-\bar{v}+\gamma \\
\bar{v}_{t} & =a \bar{u}-b \bar{v}+\mu
\end{aligned}\right.
$$

But,

$$
\begin{aligned}
|g(t)| & =\left|\frac{1}{|\Omega|} \int_{\Omega}(f(u)-f(\bar{u}))\right| \\
& \leq \frac{L}{|\Omega|} \int_{\Omega}|u-\bar{u}| \\
& \leq \frac{L}{|\Omega|^{\frac{1}{2}}} \| u-\left.\bar{u}\right|_{\mathrm{L}^{2}(\Omega)},
\end{aligned}
$$

where,

$$
L=\sup _{t \in \mathbb{R}^{+}}\left|f^{\prime}(\bar{u}(t))\right|,
$$

since from a result in [6], we know that $(u, v) \in L^{\infty}(\Omega) \times L^{\infty}(\Omega)$. It follows that :

$$
\lim _{t \rightarrow+\infty} g(t)=0 .
$$

Which completes the proof.

Let $\left(u_{i}, v_{i}\right), 1 \leq i \leq N$ be the solution of system (3)

Théorème 2.2. Let $\lambda$ be the smallest non-zero eigenvalue of the Laplacian operator, with zero flux Neumann boundary conditions. Assume that,

$$
M-\lambda d_{u_{1}}<0 \text { and } M-\lambda d_{u_{i}}-\alpha_{i}<0 \quad \forall i \in 2, . ., N,
$$

then,

$$
\lim _{t \rightarrow+\infty} \sum_{i=1}^{N}\left(\left\|u_{i}-\bar{u}_{i}\right\|_{\mathrm{L}^{2}(\Omega)}+\left\|v_{i}-\bar{v}_{i}\right\|_{\mathrm{L}^{2}(\Omega)}\right)=0,
$$

where,

with $\left(\bar{u}_{i}, \bar{v}_{i}\right)$ satisfying,

$$
\bar{u}_{i}(t)=\frac{\int_{\Omega} u_{i}(x, t) d x}{|\Omega|}, \quad \bar{v}_{i}(t)=\frac{\int_{\Omega} v_{i}(x, t) d x}{|\Omega|}, \quad \forall i \in 1, \ldots, N
$$




$$
\left\{\begin{aligned}
\epsilon \bar{u}_{i t} & =f\left(\bar{u}_{i}\right)-\bar{v}_{i}+\gamma_{i}+g_{i}(t)+\alpha_{i}\left(\bar{u}_{i-1}-\bar{u}_{i}\right) \\
\bar{v}_{i t} & =a_{i} \bar{u}_{i}-b_{i} \bar{v}_{i}+\mu_{i}+\beta_{i}\left(\bar{v}_{i-1}-\bar{v}_{i}\right)
\end{aligned}\right.
$$

and where, $g_{i}(t) \rightarrow 0$ when $t \rightarrow+\infty$ with exponential rate decay.

Démonstration. It comes from an induction argument, by using similar techniques as those given in the proof of Theorem 2.1. More precisely, let,

$$
\phi_{i}=\frac{1}{2}\left(\epsilon a_{i} \int_{\Omega}\left|\nabla u_{i}\right|^{2}+\int_{\Omega}\left|\nabla v_{i}\right|^{2}\right),
$$

we show that for all $i \in 1, \ldots, N$ there exists positive constants $K_{i}, c_{i}$ such that,

$$
\phi_{i}(t) \leq K_{i} e^{-c_{i} t}
$$

From the proof of Theorem 2.1, we know that this result is true for $i=1$, that is,

$$
\phi_{1}(t) \leq \phi_{1}(0) e^{-c_{1} t}
$$

Let us assume that the result is true until $i-1$, by algebraic computations we obtain,

$$
\begin{aligned}
\dot{\phi}_{i} & \leq a_{i}\left(M-\lambda d_{u_{i}}-\alpha_{i}+\frac{\alpha_{i} \kappa_{i}}{2}\right) \int_{\Omega}\left|\nabla u_{i}\right|^{2}-\left(\lambda d_{v_{i}}+b_{i}+\frac{\beta_{i}}{2}\right) \int_{\Omega}\left|\nabla v_{i}\right|^{2}+a_{i} \frac{\alpha_{i}}{2 \kappa_{i}} \int_{\Omega}\left|\nabla u_{i-1}\right|^{2}+\frac{\beta_{i}}{2} \int_{\Omega}\left|\nabla v_{i-1}\right|^{2} \\
& \leq a_{i}\left(M-\lambda d_{u_{i}}-\alpha_{i}+\frac{\alpha_{i} \kappa_{i}}{2}\right) \int_{\Omega}\left|\nabla u_{i}\right|^{2}-\left(\lambda d_{v_{i}}+b_{i}+\frac{\beta_{i}}{2}\right) \int_{\Omega}\left|\nabla v_{i}\right|^{2}+s_{1} K_{i-1} e^{-c_{i-1} t} \\
& \leq-s_{2} \phi+K_{i-1} e^{-c_{i-1} t}
\end{aligned}
$$

where $\kappa_{i}$ is a positive constant satisfying

$$
\kappa_{i}<2 \frac{\lambda d_{u_{i}}+\alpha_{i}-M}{\alpha_{i}}
$$

and $s_{1}=\max \left(\frac{\alpha_{i}}{\epsilon \kappa_{i}}, \beta_{i}\right), s_{2}=2 \min \left(\frac{\lambda d_{u_{i}}+\alpha_{i}\left(1-\frac{\kappa_{i}}{2}\right)-M}{\epsilon}, \lambda d_{v_{i}}+b_{i}+\frac{\beta_{i}}{2}\right), K_{i-1}, c_{i-1}$ are positive constants. By integration, this yields,

$$
\phi_{i}(t) \leq K_{i} e^{-c_{i} t}
$$

The remaining of the proof is similar as this of Theorem 2.1.

\subsection{Synchronization}

Définition 2.3. Let $S_{i}=\left(u_{i}, v_{i}\right)$. We say that $S_{i}$ and $S_{j}$ synchronize if,

$$
\lim _{t \rightarrow+\infty}\left(\left\|u_{i}-u_{j}\right\|_{L^{2}(\Omega)}+\left\|v_{i}-v_{j}\right\|_{L^{2}(\Omega)}\right)=0 .
$$

The quantity,

$$
\left(\left\|u_{i}-u_{j}\right\|_{\mathrm{L}^{2}(\Omega)}^{2}+\left\|v_{i}-v_{j}\right\|_{\mathrm{L}^{2}(\Omega)}^{2}\right)^{\frac{1}{2}}
$$


is called the norm of synchronization error between $S_{i}$ and $S_{j}$. Let $S=\left(S_{1}, S_{2}, \ldots, S_{N}\right)$. We say that $S$ synchronize if,

$$
\lim _{t \rightarrow+\infty} \sum_{i=1}^{N-1}\left(\left\|u_{i}-u_{i+1}\right\|_{L^{2}(\Omega)}+\left\|v_{i}-v_{i+1}\right\|_{L^{2}(\Omega)}\right)=0
$$

The quantity,

$$
\left(\sum_{i=1}^{N-1}\left(\left\|u_{i}-u_{i+1}\right\|_{\mathrm{L}^{2}(\Omega)}^{2}+\left\|v_{i}-v_{i+1}\right\|_{\mathrm{L}^{2}(\Omega)}^{2}\right)\right)^{\frac{1}{2}}
$$

is called the norm of synchronization error of $S$.

Let us consider the system (3) with $d_{u_{i}}=d_{u_{j}}, d_{v_{i}}=d_{v_{j}}$ and $b_{i}=b_{j}=b, a_{i}=a_{j}=a, \gamma_{i}=\gamma_{j}, \mu_{i}=\mu_{j}$, for all $i, j \in\{1, \ldots, N\}$. Let us recall that $f$ is a polynomial function of odd degree with negative leading coefficient,

$$
f(u)=\sum_{k=1}^{p} d_{k} u^{k}, d_{p}<0, p \geq 3 .
$$

Let,

$$
M=\sup _{u \in B, x \in \mathbb{R}} \sum_{k=1}^{p} \frac{f^{(k)}}{k !}(u) x^{k-1},
$$

where $B$ is a compact interval in which $u_{1}$ remains strictly.

Théorème 2.4. If,

$$
\alpha_{i}>M, \quad i=2, \ldots, N
$$

then the network $S=\left(\left(u_{1}, v_{1}\right),\left(u_{2}, v_{2}\right), \ldots,\left(u_{N}, v_{N}\right)\right)$ synchronize in the sense of definition (2.3).

Démonstration. Let

$$
\psi_{i}(t)=\frac{1}{2}\left(a \epsilon \int_{\Omega}\left(u_{i}-u_{i-1}\right)^{2}+\int_{\Omega}\left(v_{i}-v_{i-1}\right)^{2}\right) .
$$

Our proof is based on an induction idea. We show that for all $i \in 2, \ldots, N$,

$$
\psi_{i}(t) \leq K_{i} e^{-c_{i} t}
$$

We first consider the subsystem $\left(u_{2}, v_{2}\right)$. By derivating $\psi_{2}$ and using Green formula, we obtain,

$$
\begin{aligned}
\dot{\psi}_{2}(t) & \leq \int_{\Omega}\left(a\left(f\left(u_{2}\right)-f\left(u_{1}\right)-\alpha_{2}\left(u_{2}-u_{1}\right)\right)\left(u_{2}-u_{1}\right)-\left(b+\beta_{2}\right)\left(v_{2}-v_{1}\right)^{2}\right) \\
& \leq \int_{\Omega}\left(a\left(f^{\prime}\left(u_{1}\right)-\alpha_{2}+\sum_{k=2}^{p} \frac{f^{(k)}\left(u_{1}\right)}{k !}\left(u_{2}-u_{1}\right)^{k-1}\right)\left(u_{2}-u_{1}\right)^{2}-\left(b+\beta_{2}\right)\left(v_{2}-v_{1}\right)^{2}\right), \\
& \leq a\left(M-\alpha_{2}\right) \int_{\Omega}\left(u_{2}-u_{1}\right)^{2}-\left(b+\beta_{2}\right) \int_{\Omega}\left(v_{2}-v_{1}\right)^{2}
\end{aligned}
$$

this yields,

$$
\dot{\psi}_{2}(t) \leq-c_{2} \psi .
$$

where $c_{2}=\min \left(\frac{\alpha_{2}-M}{\epsilon}, b+\beta_{2}\right)$ is a positive constant. Thus, we obtain,

$$
\psi_{2} \leq \psi_{2}(0) e^{-c_{2} t}
$$


Assume the result true until $i-1$, then by algebraic computations we obtain,

$$
\begin{aligned}
\dot{\psi}_{i}(t) \leq & \int_{\Omega}\left(a\left(f\left(u_{i}\right)-f\left(u_{i-1}\right)-\alpha_{i}\left(u_{i}-u_{i-1}\right)+\alpha_{i-1}\left(u_{i-1}-u_{i-2}\right)\right)\left(u_{i}-u_{i-1}\right)\right. \\
& \left.-\left(b+\beta_{i}\right)\left(v_{i}-v_{i-1}\right)^{2}+\beta_{i-1}\left(v_{i-1}-v_{i-2}\right)\left(v_{i}-v_{i-1}\right)\right) \\
\leq & \int_{\Omega}\left(a\left(M-\alpha_{i}\right)\left(u_{i}-u_{i-1}\right)^{2}+a \alpha_{i-1}\left(u_{i-1}-u_{i-2}\right)\left(u_{i}-u_{i-1}\right)\right. \\
& \left.-\left(b+\beta_{i}\right)\left(v_{i}-v_{i-1}\right)^{2}+\beta_{i-1}\left(v_{i}-v_{i-1}\right)\left(v_{i-1}-v_{i-2}\right)\right) \\
\leq & \int_{\Omega}\left(a\left(M-\alpha_{i}\right)\left(u_{i}-u_{i-1}\right)^{2}+\frac{a}{2}\left(\frac{\alpha_{i-1}^{2}}{\alpha_{i}-M}\left(u_{i-1}-u_{i-2}\right)^{2}+\left(\alpha_{i}-M\right)\left(u_{i}-u_{i-1}\right)^{2}\right)\right. \\
& \left.-\left(b+\beta_{i}\right)\left(v_{i}-v_{i-1}\right)^{2}+\frac{1}{2}\left(\frac{\beta_{i-1}^{2}}{\beta_{i}}\left(v_{i-1}-v_{i-2}\right)^{2}+\beta_{i}\left(v_{i}-v_{i-1}\right)^{2}\right)\right) \\
\leq & \int_{\Omega}\left(a \frac{M-\alpha_{i}}{2}\left(u_{i}-u_{i-1}\right)^{2}-\left(b+\frac{\beta_{i}}{2}\right)\left(v_{i}-v_{i-1}\right)^{2}\right. \\
& \left.+\frac{a}{2} \frac{\alpha_{i-1}^{2}}{\alpha_{i}-M}\left(u_{i-1}-u_{i-2}\right)^{2}+\frac{\beta_{i-1}^{2}}{2 \beta_{i}}\left(v_{i-1}-v_{i-2}\right)^{2}\right) \\
\leq & -s_{1} \psi_{i}+s_{2} K_{i-1} e^{-c_{i-1} t} .
\end{aligned}
$$

where $s_{1}=\min \left(\frac{\alpha_{i}-M}{\epsilon}, 2 b+\beta_{i}\right)$ and $s_{2}=\max \left(\frac{\alpha_{i-1}^{2}}{\epsilon\left(\alpha_{i}-M\right)}, \frac{\beta_{i-1}^{2}}{\beta_{i}}\right)$.

Then, we obtain the result by integration.

Corollaire 2.5. Assume that $f$ is a cubic function, $f(u)=d_{3} u^{3}+d_{2} u^{2}+d_{1} u$ with $d_{3}<0$. If,

$$
\alpha_{i}>d_{1}-\frac{d_{2}^{2}}{2 d_{3}}, \quad i=2, \ldots, N
$$

then the network $S=\left(\left(u_{1}, v_{1}\right),\left(u_{2}, v_{2}\right), \ldots,\left(u_{N}, v_{N}\right)\right)$ synchronize in the sense of definition $(2.3)$.

Démonstration. In this case, by computation, we obtain that,

$$
M \leq d_{1}-\frac{d_{2}^{2}}{2 d_{3}}
$$

\section{Numerical SImUlations}

We consider the system (3) for $N=3$ with for all $i \in\{1,2,3\}, d_{u_{i}}=d_{v_{i}}=1, a_{i}=1, b_{i}=0.4$. Moreover for $i \in\{2,3\}$, we fix $\beta_{i}=0, \alpha_{i}>0$ and $\epsilon=0.1$. Thus, we consider the following network of three coupled generalized FHN systems,

$$
\left\{\begin{aligned}
\epsilon u_{1 t} & =f\left(u_{1}\right)-v_{1}+\Delta u_{1} \\
v_{1 t} & =a u_{1}-b v_{1}+\Delta v_{1} \\
\epsilon u_{2 t} & =f\left(u_{2}\right)-v_{2}+\Delta u_{2}+\alpha_{2}\left(u_{1}-u_{2}\right) \\
v_{2 t} & =a u_{2}-b v_{2}+\Delta v_{2} \\
\epsilon u_{3 t} & =f\left(u_{3}\right)-v_{3}+\Delta u_{3}+\alpha_{3}\left(u_{2}-u_{3}\right) \\
v_{3 t} & =a u_{3}-b v_{3}+\Delta v_{3}
\end{aligned}\right.
$$




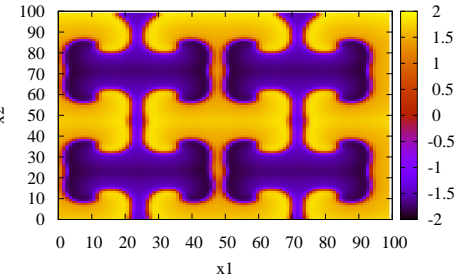

(a)

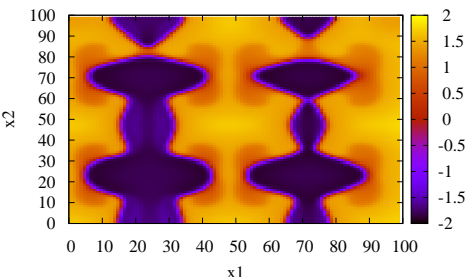

(b)

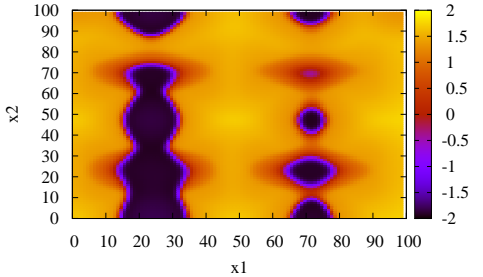

(c)

FIGURE 1. Network of three systems of generalized FHN type. Isovalues, of $(a) u_{1}(x, t)$, (b) $u_{2}(x, t),(c) u_{3}(x, t)$ at fixed time $t=190$ for the coupling strength $\alpha_{2}=\alpha_{3}=0.3$.

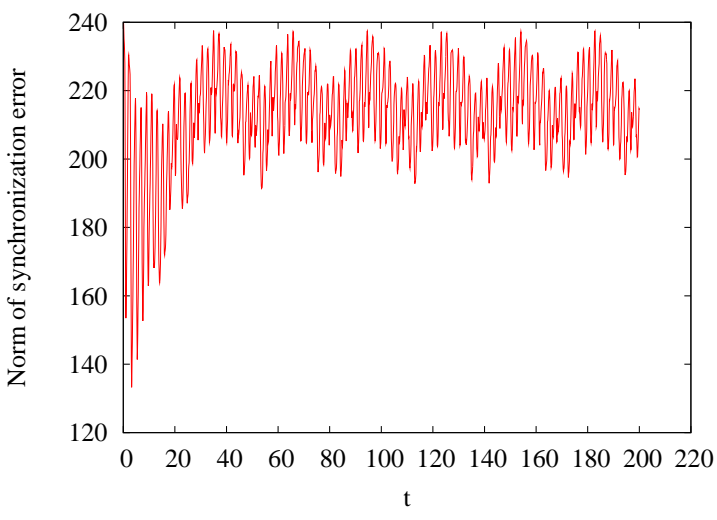

(a)

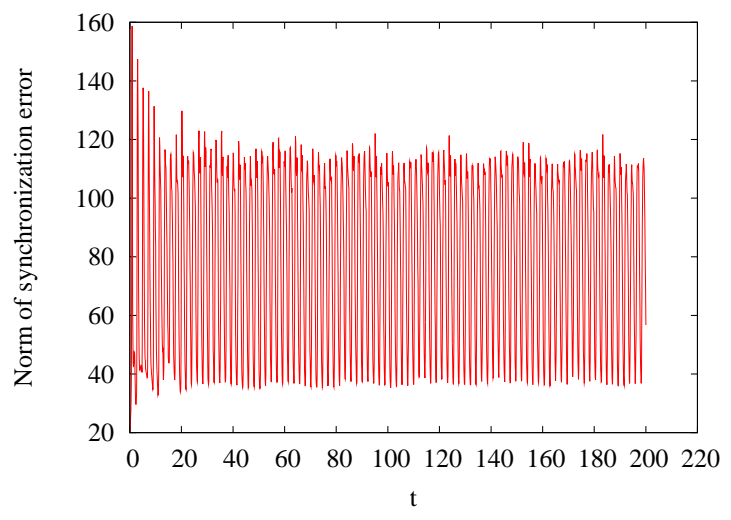

(b)

FiguRE 2. Network of three systems of generalized FHN type. The norm of synchronization error given by the definition 2.3 on the interval of time $[0,200]$ for the coupling strength $\alpha_{2}=$ $\alpha_{3}=0.3:(a)$ between $S_{1}$ and $S_{2},(b)$ between $S_{2}$ and $S_{3}$.

Our numerical simulations, see figure 1, 2, 3, 4, show that system (11) synchronize for a coupling strength $\alpha_{2}=\alpha_{3}$ belonging to the interval [0.3,0.4]. In these figures, the initial conditions are $\left(u_{1}(x, 0), v_{1}(x, 0)\right)$, particular functions leading to multiple spiral pattern formation, see [8,9], and $\left(u_{2}(x, 0), v_{2}(x, 0)\right)=\left(u_{3}(x, 0), v_{3}(x, 0)\right)=1$. Numerical simulations have been performed using an explicit finite difference scheme, with $\mathrm{C}++$ language and with a time step discretization equal to 0.01 and space step discretization equal to 1 . 


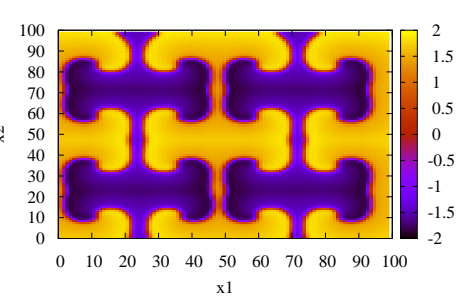

(a)

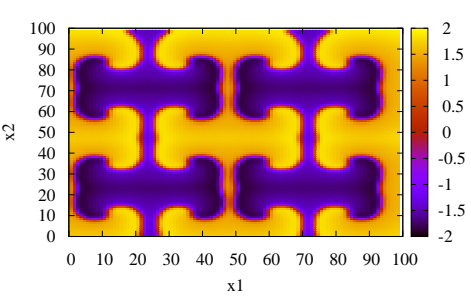

(b)

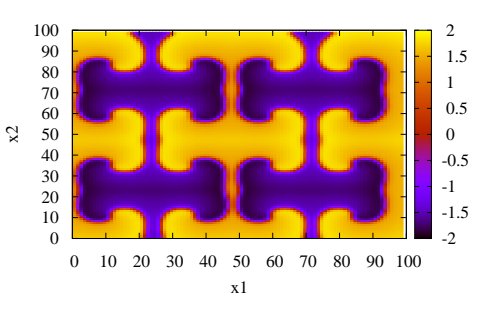

(c)

FiguRE 3. Network of three systems of generalized FHN type. Isovalues, of $(a) u_{1}(x, t)$, (b) $u_{2}(x, t),(c) u_{3}(x, t)$ at fixed time $t=190$ for the coupling strength $\alpha_{2}=\alpha_{3}=0.4$.

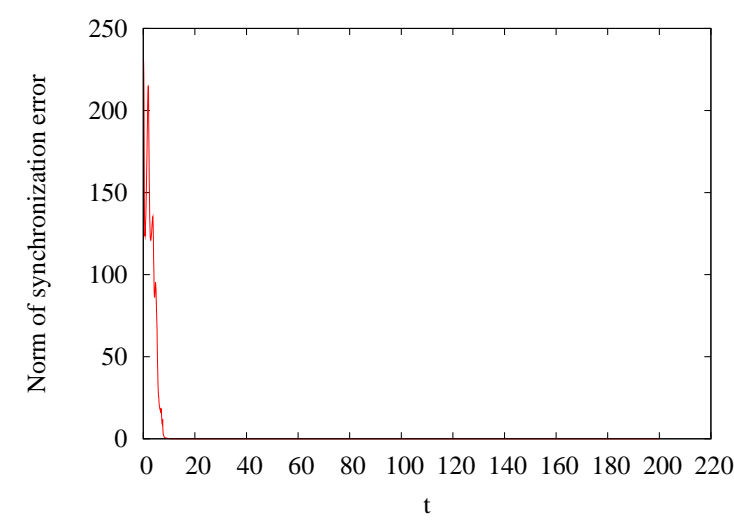

(a)

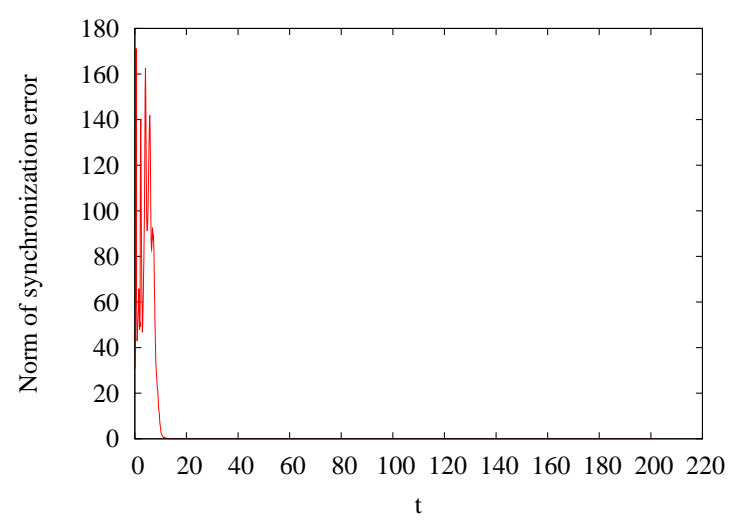

(b)

FigurE 4. Network of three systems of generalized FHN type. The norm of synchronization error given by the definition 2.3 on the interval of time $[0,200]$ for the coupling strength $\alpha_{2}=$ $\alpha_{3}=0.4:(a)$ between $S_{1}$ and $S_{2},(b)$ between $S_{2}$ and $S_{3}$. 


\section{REFERENCES}

[1] E. M. Izhikevich, Dynamical systems in Neuroscience, The MIT Press, 2007.

[2] J. P. Keener and J. Sneyd, Mathematical Physiology, Springer, 2009.

[3] J.D. Murray, Mathematical Biology, Springer, 2010.

[4] R. A. FitzHugh, Impulses and physiological states in theoretical models of nerve membrane, Biophys. J. 1, (1961) 445-466.

[5] A.L. Hodgkin and A.F. Huxley, A quantitative description of membrane current and its application to conduction and excitation in nerve, J. Physiol. 117, (1952) 500-544.

[6] M. Marion, Finite-Dimensionnal attractors associated with partly dissipative reaction-diffusion systems, SIAM J. Math. Anal. 20 (1989) 816-844.

[7] Nagumo J., Arimoto S. and Yoshizawa S, An active pulse transmission line simulating nerve axon, Proc. IRE. 50 (1962) 2061-2070.

[8] B. Ambrosio, Wave propagation in excitable media : numerical simulations and analytical study, in french. Ph.D Thesis, University Paris VI, 2009.

[9] B. Ambrosio and M.A. Aziz-Alaoui, Synchronization and control of coupled reaction-diffusion systems of the FitzHugh-Nagumo type, CAMWA. 64 (2012) 934,943.

[10] E. Conway and D. Hoff and J. Smoller, Large-time behaviour of solutions of systems of non linear reaction-diffusion equations, SIAM J. Appl. Math. 35 (1978) 1-16. 